\title{
Évaluation des substrats et des plants produits en pépinière forestière
}

\section{Youssef M'SADAK}

Mohamed Aymen Elouaer

Rim El KAMEL

Université de Sousse

Institut supérieur agronomique

de Chott Mériem (ISA-CM)

BP 47, 4042 Chott Mériem

Sousse

Tunisie

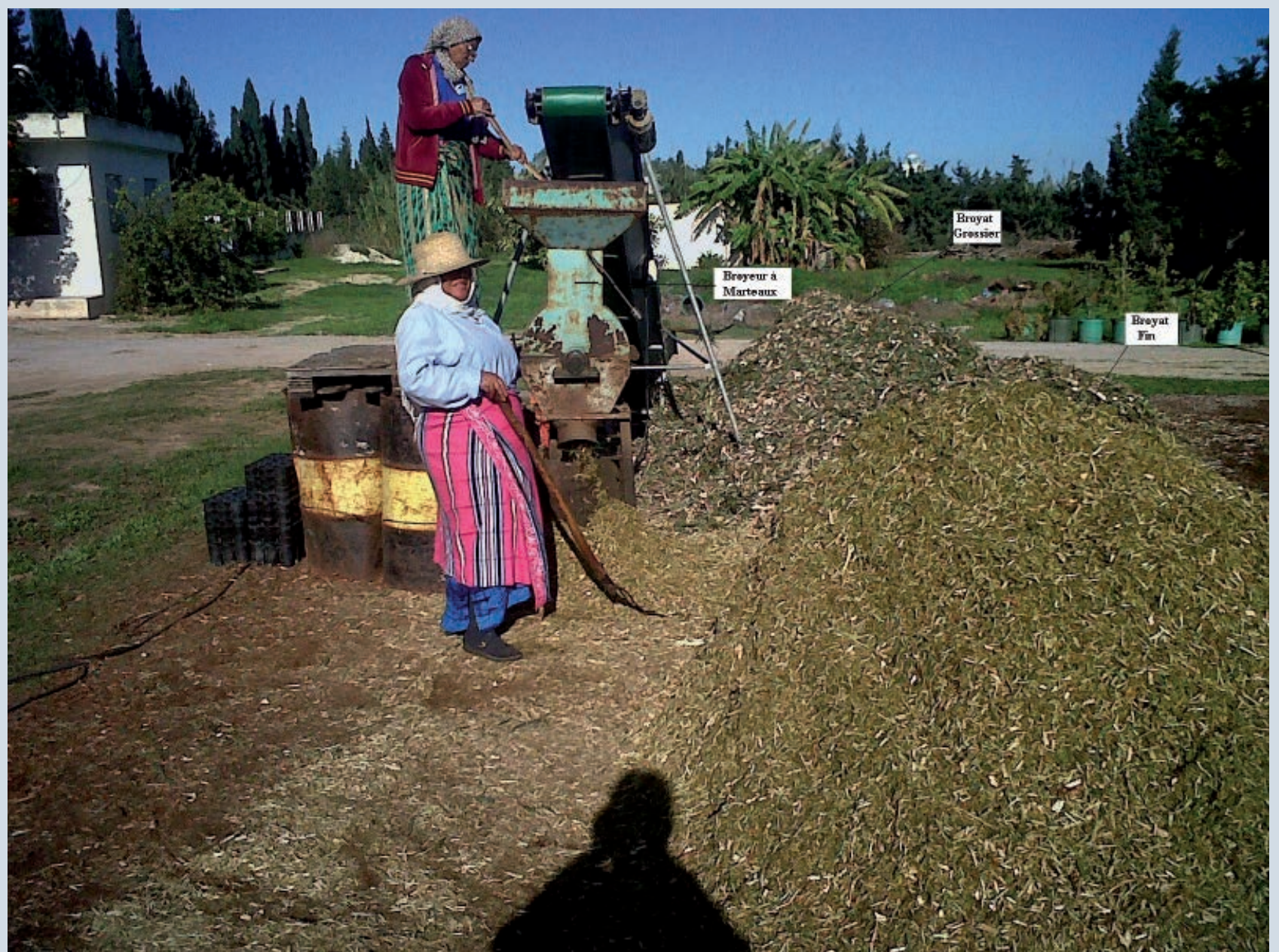

Photo 1.

Aire de broyage des branches d'Acacia cyanophylla en pépinière.

Photo M. A. Elouaer. 


\section{RÉSUMÉ}

\section{ÉVALUATION DES SUBSTRATS ET DES PLANTS PRODUITS EN PÉPINIĖRE FORESTIÈRE}

Le compostage représente une stratégie efficace pour recycler la biomasse sylvicole afin de confectionner des substrats de croissance adéquats pour la production des plants forestiers. Suite au processus de compostage de sept mois, les déchets sylvicoles ont atteint un degré de maturité en termes d'absence de phytotoxicité (déterminé selon le biotest de germination). Le compost ainsi obtenu est utilisé afin d'élaborer six substrats de croissance selon différentes granulométries. Les résultats obtenus montrent que le type de criblage du compost (simple criblage, double criblage sur refus ou double criblage sur tamisat) agit significativement sur les caractéristiques relevées sur le plan physique (porosités totale, d'aération et de rétention) et sur le plan chimique ( $\mathrm{pH}$, conductivité électrique, teneurs en matière organique, azote, phosphore et potassium) du substrat, et par conséquent, sur les comportements germinatif (pourcentage cumulé de germination) et végétatif (hauteur, diamètre et ratio de robustesse) des plants d'Acacia cyanophylla produits en conteneurs sur les divers substrats étudiés. Le substrat préférable de culture est le tamisat issu du double criblage aux mailles carrées $12 \times 12 \mathrm{~mm}$ et $8 \times 8 \mathrm{~mm}$ dans le contexte expérimental choisi.

Mots-clés : compost sylvicole, tamisat, substrat de croissance, Acacia cyanophylla, caractérisation physico-chimique, comportements germinatif et végétatif.

\section{ABSTRACT}

\section{ASSESSMENT OF GROWTH SUBSTRATES AND THE FOREST NURSERY PLANTS OBTAINED}

Composting is an efficient way of producing suitable growth substrates for the production of forest saplings by recycling sylvicultural biomass. After 7 months, the sylvicultural waste has decomposed sufficiently to eliminate any phytotoxicity (as determined by germination biotests). The compost obtained was used to produce substrates of six different grades in terms of granulometric composition. The results show that the screening method used (single pass, second pass to screen the material initially sifted out or second pass to screen the sifted material twice) had a significant impact on the physical characteristics of the substrate obtained (total porosity, aeration and retention capacity) as well as its chemical characteristics ( $\mathrm{pH}$, electrical conductivity, content of organic matter, nitrogen, phosphorus and potassium), and consequently, on the germination (total germination percentage) and plant growth patterns (height, diameter and robustness coefficient) of the Acacia cyanophylla saplings grown in containers on the different substrates studied. In the chosen experimental context, the most suitable substrate was produced with material screened twice, first through a $12 \times 12 \mathrm{~mm}$ mesh and again through an $8 \times 8 \mathrm{~mm}$ mesh.

Keywords: sylviculural compost, sifted material, substrate, Acacia cyanophylla, physico-chemical characterisation, germination and growth patterns.

\section{RESUMEN}

\section{EVALUACIÓN DE SUSTRATOS Y PLANTAS PRODUCIDOS EN VIVERO FORESTAL}

El compostaje representa una estrategia eficaz para reciclar la biomasa forestal con el fin de elaborar sustratos de crecimiento adecuados para la producción de plantas forestales. Tras un proceso de compostaje de 7 meses, los residuos forestales han alcanzado un grado de madurez en términos de ausencia de fitotoxicidad (determinado mediante bioensayo de germinación). El compost así obtenido es utilizado para elaborar seis sustratos de crecimiento con diferentes granulometrías. Los resultados obtenidos muestran que el tipo de cribado del compost (cribado simple, doble cribado sobre rechazo o doble cribado sobre tamizado) influye significativamente en las características estudiadas del sustrato, tanto en el aspecto físico (porosidad total, de aireación y de retención) como en el químico ( $\mathrm{pH}$, conductividad eléctrica, contenidos en materia orgánica, nitrógeno, fósforo y potasio) y, por consiguiente, influye en el comportamiento germinativo (porcentaje acumulado de germinación) y vegetativo (altura, diámetro y coeficiente de robustez) de plantas de Acacia cyanophylla producidas en contenedores en los distintos sustratos estudiados. El sustrato más conveniente para el cultivo es el tamizado obtenido mediante doble cribado de la fracción pasante por malla cuadrada $(12 \times 12 \mathrm{~mm})$ y por malla cuadrada $(8 \times 8 \mathrm{~mm})$ en el contexto experimental ensayado.

Palabras clave: compost forestal, tamizado, sustrato de crecimiento, Acacia cyanophylla, caracterización fisicoquímica, comportamiento germinativo y vegetativo. 


\section{Introduction}

Plusieurs pépinières forestières rencontrent des obstacles d'approvisionnement en terreau forestier de manière de plus en plus permanente. Face à cette situation, elles ont généralement recours à l'emploi d'un substrat d'origine minérale dont les propriétés physico-chimiques influencent négativement la croissance des plants. En plus, le terreau forestier constitue une source d'agents pathogènes qui affectent négativement la qualité des plants produits en pépinière, ainsi que leur performance en site de reboisement (LAMHAMEDI, FoRTIN, 1994). Pour pallier ce problème, le recours au compostage constitue actuellement une alternative intéressante de remplacement du terreau forestier par le compost dans la confection des substrats de culture. En effet, le compostage permet la décomposition biologique et la stabilisation des substrats organiques (HAUG, 1993 ; Miller, Jones, 1995 ; Stofella, Kahn, 2001). Le compostage des déchets sylvicoles et agricoles a donné d'excellents résultats en matière de fabrication des substrats pour produire des plants en pépinières horticoles et forestières (Lemaire et al., 1989 ; LANDIS, 1990 ; MILLER, JONES, 1995 ; Rose et al., 1995 ; FITZPATRICK, 2001).

Divers travaux ont démontré les possibilités d'utilisation des composts dans la production des plants forestiers (MILLER, JONES, 1995 ; RAVIV et al., 1998 ; FITZPATRICK, 2001). L'optimisation des techniques culturales en pépinières forestières et horticoles (irrigation, fertilisation, traitements phytosanitaires, etc.) ne peut à elle seule garantir une production de plants de qualité si les propriétés physico-chimiques du substrat de culture ne sont pas satisfaisantes (GUEHL et al., 1989 ; LANDIS et al., 1990 ; ALSANIUS et al., 2004). Les études de VERDONCK et al. (1983) suggèrent que n'importe quel substrat de croissance doit avoir une capacité de rétention en air de $20 \%$ et celle en eau entre 20 et $30 \%$ en volume. L'ajustement de la taille des particules du substrat de croissance par différentes techniques et méthodes de criblage afin d'accommoder les porosités nécessaires fait débat dans plusieurs recherches scientifiques (REISCH, 1967 ; BILDERBACK et al., 1982 ; BugBEE, FrInK, 1986 ; NKONGOLO, CARON, 1999).
De même, plusieurs travaux de recherche ont montré que la taille des particules du substrat a une influence sur la croissance des plants ; en effet, RICHARDS et al. (1986) ont trouvé que les plants de tomate et Boronia nécessitent un substrat dont la taille des particules est comprise entre $10 \mathrm{~mm}$ et $4,75 \mathrm{~mm}$; cependant, cette taille est inférieure à $5 \mathrm{~mm}$ pour les plantes forestières du genre Peperomia. Tilt et al. (1987) ont constaté que la croissance des plantes forestières et ornementales (Leyland cypress, azalea et holly) a été meilleure lorsque la taille des particules présentes dans le substrat est de l'ordre de 3 à $5 \mathrm{~mm}$.

C'est à partir de ces constatations que le présent travail se propose comme objectif principal, d'une part, d'évaluer directement l'effet de l'ajustement de la granulométrie du substrat de croissance par criblage, en comparant six substrats à base de compost sylvicole criblé (ou tamisat) du point de vue des caractéristiques physiques et chimiques, et, d'autre part, de les évaluer indirectement en ayant recours au suivi du comportement des plants d'Acacia cyanophylla installés en pépinière forestière sur ces divers substrats.

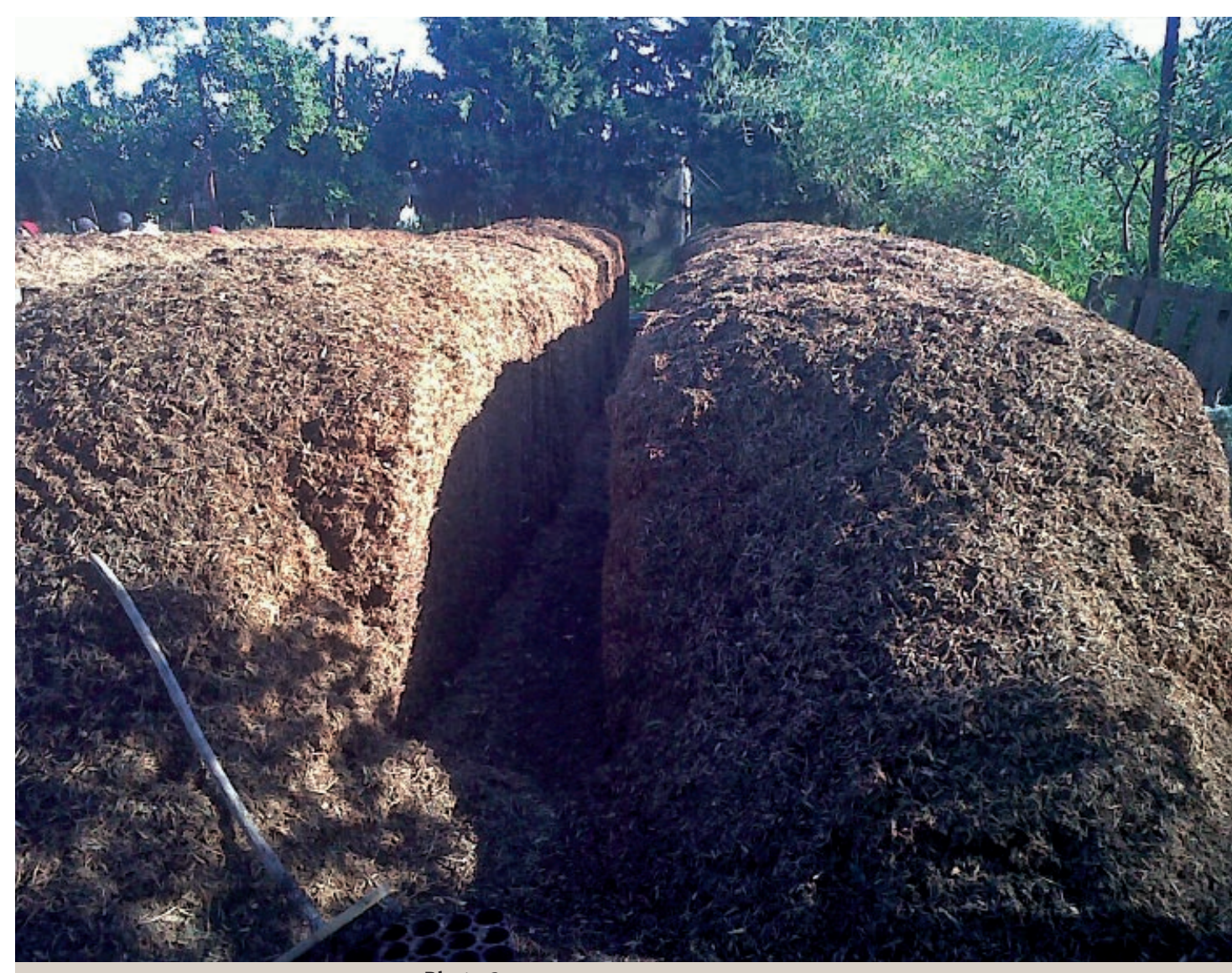

Photo 2.

Vue générale de l'aire de compostage sylvicole en pépinière. Photo M. A. Elouaer. 


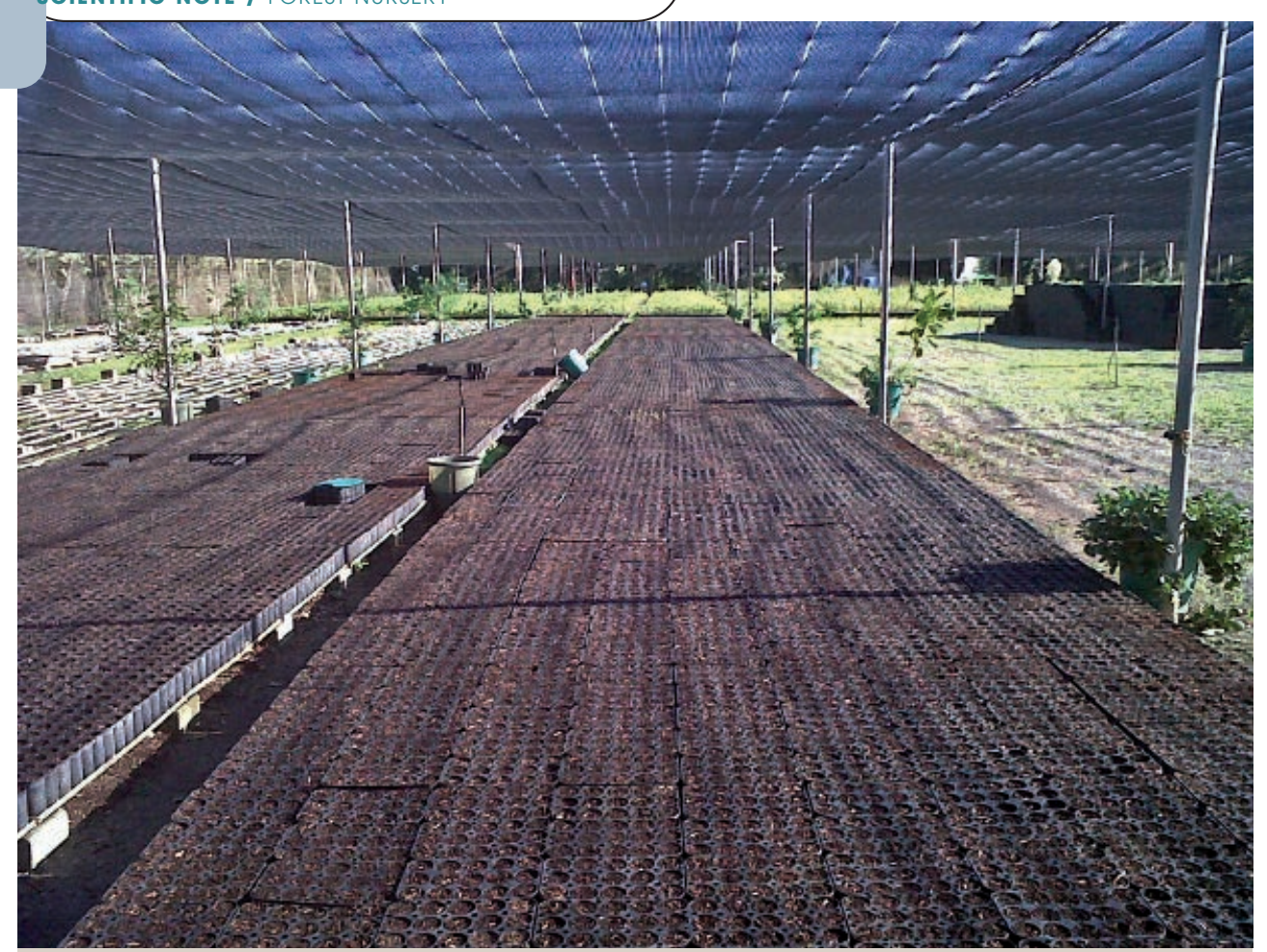

Photo 3.

Aire de culture des plants d'Acacia cyanophylla sous ombrière.

Photo M. A. Elouaer.

\section{Caractéristiques des composts} sylvicoles étudiés

Trois andains de compost à base de broyat d'acacia ont été mis à l'étude (photo 2). Pour s'assurer de la maturité des différents composts considérés et de l'absence d'une toxicité avant leur utilisation comme substrat de croissance pour l'élevage des plants forestiers, des biotests à base de légumineuses (pois chiche et fève) ont été effectués sur les trois composts bruts représentatifs des trois andains considérés (CA1, CA2, CA3).

\section{Biotest de germination Mode opératoire}

Des conteneurs (15 cavités chacun) ont été remplis par le compost sylvicole brut à tester, issu des trois andains fixés. Les graines de fève et de pois chiche, préalablement imbibées dans l'eau pendant 24 heures, ont été semées à raison d'une graine par

\section{Matériel et méthodes}

\section{Compostage}

\section{Broyage de la biomasse mise en compostage}

Le compost sylvicole utilisé dans l'expérimentation mise en œuvre est à base de branches fraîches broyées d'Acacia cyanophylla. Le broyage des branches d'acacia (de diamètre inférieur à $8 \mathrm{~cm}$ ) a été effectué successivement par deux broyeurs, l'un à couteaux, assurant un premier broyage sur le lieu d'approvisionnement en donnant un broyat grossier, et l'autre à marteaux avec grille de calibrage à trous ronds de diamètre $30 \mathrm{~mm}$, garantissant un second broyage en pépinière en générant un broyat fin (photo 1). Il s’agit de la technique du double broyage séparé (couteaux-marteaux).

\section{Mise en andains et processus de compostage}

L'andainage est réalisé sur une aire bétonnée. Le broyat d'acacia est déposé en couches de matière fraîche auxquelles est ajouté le nitrate d'ammonium $\left(\mathrm{NH}_{4} \mathrm{NO}_{3}\right)$ comme source d'azote à raison de $2 \mathrm{~kg} / \mathrm{m}^{3}$ de broyat répartis en deux apports. L'eau est apportée afin d'avoir une humidité optimale de 50 à $60 \%$.

\section{Suivi de l'évolution du compost sylvicole}

Le retournement du compost est effectué en fonction de la température. Il est réalisé manuellement à la fréquence de trois à cinq par cycle de compostage (six mois au minimum). Le suivi quotidien de la température du compost s'effectue, d'une manière continue et en six endroits répartis au niveau de chaque andain, à l'aide d'un thermomètre à sonde de $90 \mathrm{~cm}$ de longueur. cavité. Pour chaque compost, on a utilisé 8 conteneurs, soit au total 24 conteneurs (12 pour le semis de fève et 12 pour le semis de pois chiche, soit au total 180 graines de fève et 180 graines de pois chiche).

\section{Variables mesurées}

Le suivi a porté sur le pourcentage cumulé de germination, cinq jours après le semis pendant 15 jours, et la croissance en hauteur des plantules, 15 jours après le début de la germination.

Suite aux relevés relatifs au biotest de germination mis en œuvre, le compost CA3 a été choisi comme étant le meilleur compost sur le plan de la maturité, car il offrait les taux de germination les plus élevés pour les plants-tests de fève et de pois chiche, évalués respectivement à $70 \%$ et $85 \%$.

\section{Substrats de croissance}

\section{Confection des substrats à base de tamisat}

Les substrats de culture testés, issus du compost CA3 de maturité jugée convenable, au nombre de six, sont les suivants :

- SA, compost sylvicole brut non criblé ;

- SB, substrat résultant d'un simple criblage à la maille carrée $6 \times 6 \mathrm{~mm}$;

- SC, substrat résultant d'un simple criblage à la maille carrée $8 \times 8 \mathrm{~mm}$;

- SD, substrat résultant d'un simple criblage à la maille carrée $12 \times 12 \mathrm{~mm}$;

- $\mathrm{SE}$, substrat consécutif à un double criblage respectivement aux mailles $8 \times 8 \mathrm{~mm}$ et $12 \times 12 \mathrm{~mm}$;

- SF, substrat consécutif à un double criblage respectivement aux mailles $12 \times 12 \mathrm{~mm}$ et $8 \times 8 \mathrm{~mm}$. 
Caractérisation physique par le test standard de porosité Mode opératoire

Pour déterminer la porosité d'un substrat, la méthode standard suivante a été utilisée :

- obstruer les trous de drainage du conteneur et le remplir avec de l'eau (volume d'eau = volume du conteneur) ;

- enlever l'eau et remplir trois cavités parmi 15 avec le substrat sec ;

- verser de l'eau très lentement sur toute la surface des cavités jusqu'à ce que l'eau apparaisse à la surface ;

- attendre environ une heure et rajouter de l'eau si nécessaire (volume d'eau versé = volume des pores : air et eau) ; - dégager les trous de drainage et recueillir l'eau qui s'écoule pendant 5 à 10 minutes (volume d'eau recueilli = volume des pores : air).

\section{Variables mesurées}

Volume total (VT) : il s'agit du volume d'eau versé dans les trois cavités.

Volume des pores (VA) : il représente le volume d'eau versé (air et eau).

Volume de la phase gazeuse (VR) : c'est le volume d'eau acquis après dégagement des trous de drainage, le volume d'eau obtenu correspondant au volume de la phase gazeuse.

\section{Paramètres étudiés}

Les paramètres de porosité sont déterminés comme suit : - porosité totale Pt $(\%)=(\mathrm{VA} / \mathrm{VT}) \times 100$;

- porosité d'aération Pa $(\%)=(V R / V T) \times 100$;

- porosité de rétention $\operatorname{Pr}(\%)=\mathrm{Pt}-\mathrm{Pa}$.

Compte tenu du climat sec de la Tunisie, les règles d'appréciation suivantes ont été retenues :

$P t \geq 50 \%, P a \geq 20 \%$ et $\operatorname{Pr} \geq 30 \%$.

\section{Caractérisation chimique}

Les paramètres chimiques mesurés sont les suivants : $\mathrm{pH}$, conductivité électrique, salinité, matière organique, azote, rapport carbone/azote, phosphore et potassium.

La mesure du $\mathrm{pH}$ est réalisée selon la norme internationale (Iso, 1994). La lecture du $\mathrm{pH}$ se fait au moyen d'un pH-mètre.

La conductivité électrique (CE) est la mesure de la concentration des ions solubles afin d'apprécier la salinité du substrat (TIQUIA, 2010). Elle est mesurée par conductimètre et elle est exprimée en $\mathrm{mS} / \mathrm{cm}$ ou en $\mathrm{mmhos} / \mathrm{cm}^{3}$. La norme internationale (Iso, 1994) prescrit une méthode pour sa mesure. Un échantillon de substrat est extrait avec de l'eau à $20 \pm 1{ }^{\circ} \mathrm{C}$ (rapport d'extraction de $1 / 5$ pour dissoudre les électrolytes).

La salinité (S) est déterminée à partir de la conductivité électrique (C.E) en utilisant l'équation suivante :

$\mathrm{S}=0,7 \times \mathrm{CE}$, avec :

- $\mathrm{CE}$, conductivité électrique, exprimée en $\mathrm{mmhos} / \mathrm{cm}^{3}$;

- S, salinité, exprimée en $\mathrm{g} / \mathrm{l}$ de sel.

Afin de déterminer le taux de matière organique (MO) suivant la norme tunisienne (NT) relative au dosage de la MO du fumier, la méthode considérée, pour la détermination de ce taux au sein de chaque substrat, comporte les deux étapes suivantes:

- une quantité de $20 \mathrm{~g}$ de chaque substrat est mise dans l'étuve pendant 24 heures à $70^{\circ} \mathrm{C}$;

- une quantité de $3 \mathrm{~g}$ de chaque échantillon, préalablement séchée pendant 2 heures à l'étuve, est calcinée à $900^{\circ} \mathrm{C}$ pendant 6 heures dans un four à moufle. La teneur en MO est déterminée selon l'équation suivante :

$\mathrm{MO}(\%)=((\mathrm{M} 1-\mathrm{M} 2) / \mathrm{M} 1) \times 100$,

avec :

- M1, masse avant calcination (mg) ;

- M2, masse après calcination (mg).

À partir de la MO, la teneur du carbone organique total (COT) est déduite selon la relation suivante :

COT $(\%)=(M O(\%) / 1,8) \times 100$.

L'azote (N) est dosé par la méthode de Kjeldhal (GoyAL et al., 2005).

Le dosage du phosphore total $(P)$ s'effectue par spectrométrie d'absorption atomique. Il est effectué en présence d'un réactif vitrovanadomolybdate.

L'acide phosphorique donne un complexe phosphomolybdique jaune dont la densité optique est mesurée par spectrophotométrie à $430 \mathrm{~nm}$.

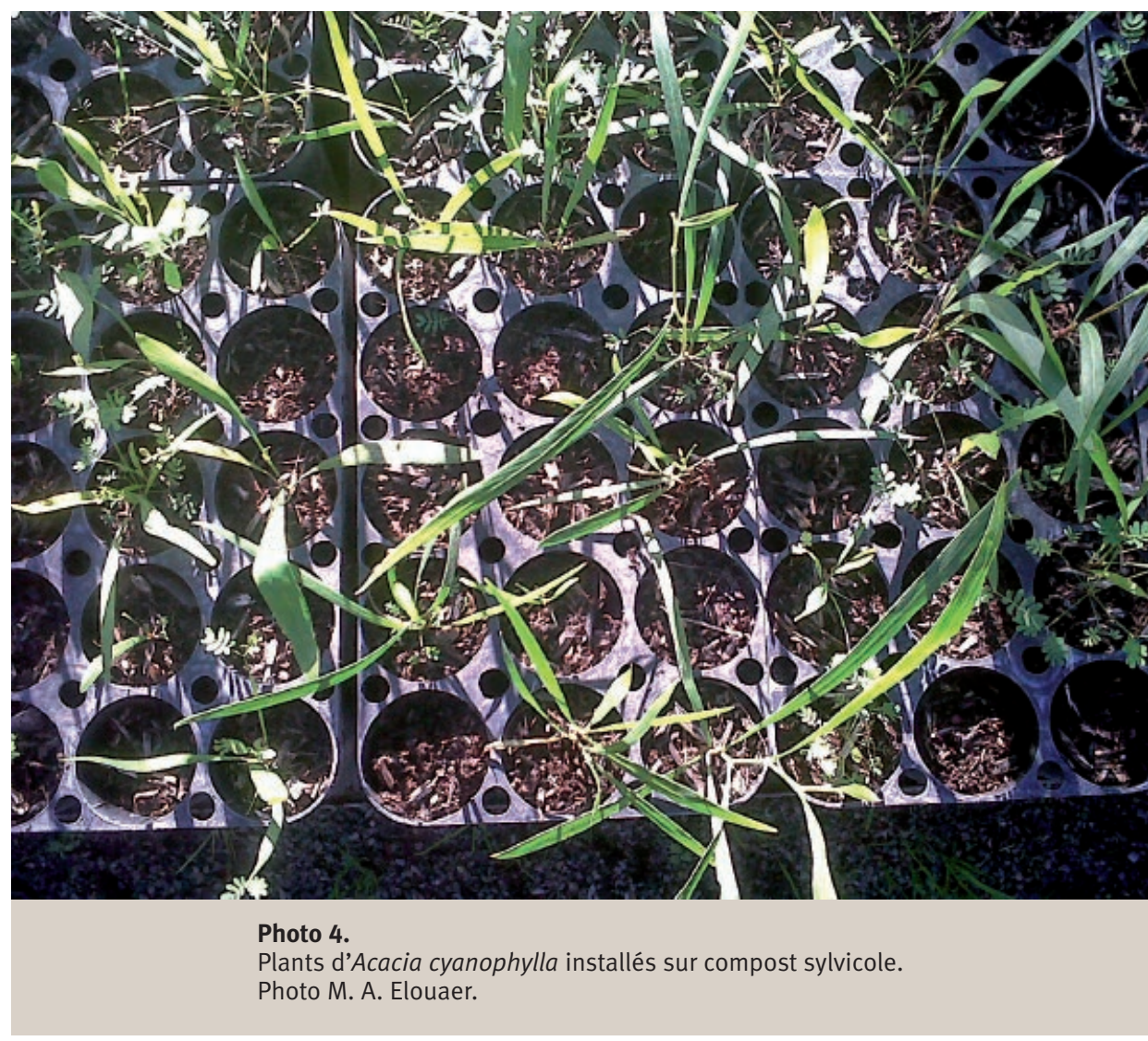




\section{Résultats et discussion}

Après la calcination des échantillons dans le four à moufle, l'eau distillée est ajoutée pour obtenir une solution saline de cendres. Dans une fiole jaugée de $25 \mathrm{ml}$, on prélève $10 \mathrm{ml}$ de la solution saline déjà préparée. Une quantité de $5 \mathrm{ml}$ de réactif nitrovanadomolybdique est ajoutée et complétée jusqu'au trait de jauge avec de l'eau distillée. Après 1 heure d'attente, la solution est passée au photocolorimètre.

Le dosage du potassium (K) a été réalisé à l'aide d'un photomètre à flamme. Une quantité de $1 \mathrm{~g}$ de chaque substrat est calcinée dans un four à moufle pendant 2 heures à une température de $220^{\circ} \mathrm{C}$ et 6 heures à une température de $550^{\circ} \mathrm{C}$. Après refroidissement, $2 \mathrm{ml}$ d'acide chlorhydrique $(\mathrm{HCl})$ sont ajoutés. Les substrats sont chauffés dans un bain à sable jusqu'à l'évaporation totale de l'acide. Un volume de $5 \mathrm{ml}$ de $\mathrm{HCl}(\mathrm{N} / 10)$ y est ajouté et ensuite les solutions obtenues sont filtrées dans des fioles jaugées de $100 \mathrm{ml}$ et de l'eau distillée est ajoutée jusqu'au trait de jauge. Les échantillons sont passés dans le photomètre à flamme après avoir passé les solutions d'étalonnage appropriées à chaque élément minéral.

\section{Comportement des jeunes plants d'Acacia cyanophylla}

L'objectif est ici d'étudier le comportement des jeunes plants d'Acacia cyanophylla vis-à-vis de six substrats à base de tamisat sylvicole (SA, SB, SC, SD, SE et SF), afin de tester l'influence de la taille des particules sur la germination des semences et le développement des plants produits en conteneurs placés sur des tables de culture sous ombrière (photo 3).

Les paramètres morphologiques étudiés au cours de cet essai sont le pourcentage de germination, la hauteur, le diamètre et le ratio de robustesse. Quarante-huit conteneurs ont été remplis de six substrats de croissance différents. Durant la période d'expérimentation, il a été appliqué aux jeunes plants une simple irrigation tous les deux jours jusqu'à la fin du suivi (photo 4).

Le dispositif expérimental adopté est un plan en blocs aléatoires complets, chaque bloc étant constitué de six rangées de récipients. Chaque rangée comporte deux récipients remplis avec le même substrat. Chaque conteneur contient 15 cavités, soit 30 plants par substrat et par bloc. Les six substrats étudiés ont été distribués aléatoirement à l'intérieur d'un même bloc.

\section{Analyses statistiques}

Sur les résultats des analyses physico-chimiques des substrats et des derniers suivis de germination et de croissance, nous avons réalisé des analyses de variance (Anova) et des comparaisons de moyennes par le test de Duncan en ayant recours au logiciel Spss (13.0). Les différences ont été considérées significatives au seuil de $5 \%$ (moyennes suivies de lettres différentes). Par ailleurs, une analyse en composantes principales (Acp) a été entreprise en vue de représenter dans un espace à deux dimensions, d'une part, les substrats de croissance en question et, d'autre part, les paramètres chimiques de ces substrats.

\section{Caractéristiques physiques des substrats de croissance}

La figure 1 illustre l'évolution des porosités totale, d'aération et de rétention des six substrats de croissance étudiés.

Les six substrats testés répondent tous aux règles d'appréciation des porosités totale et d'aération ; cependant, les substrats SA, SD et SE ne sont pas conformes du point de vue de la porosité de rétention (minimum $30 \%$ ). De tels résultats révèlent, à part le compost brut, que les tamisats issus d'un simple criblage à la maille $12 \times 12 \mathrm{~mm}$ et d'un double criblage successivement sur les mailles $8 \times 8 \mathrm{~mm}$ et $12 \mathrm{x}$ $12 \mathrm{~mm}$ ne sont pas physiquement adaptés. L'analyse statistique montre une différence significative entre les différents substrats. Du point de vue de la porosité totale, les substrats (SA, SE, SF) et (SB, SC, SD) ne diffèrent pas significativement entre eux. Concernant la porosité de rétention, les substrats SA, SD, SE ne diffèrent pas significativement entre eux. Finalement, en ce qui concerne la porosité d'aération, les substrats (SA, SE), (SD, SF) et (SB, SC) ne diffèrent pas significativement. Il est possible de conclure que les substrats $S B$, SC et SF ont les meilleures porosités par rapport aux autres substrats en combinant les trois porosités étudiées.

\section{Caractéristiques chimiques des substrats de croissance}

Le tableau I illustre les résultats relatifs aux paramètres chimiques des six substrats de croissance considérés.

L'étendue du pH révélée dans le tableau I montre que ce paramètre varie entre 6 et 6,7 . Les résultats relatifs au $\mathrm{pH}$ indiquent qu'il existe une différence significative entre les substrats testés. L'opération de criblage fait diminuer significativement ce paramètre. Malgré cette diminution, le $\mathrm{pH}$ des divers échantillons est favorable pour l'assimilation des éléments nutritifs; de plus, les valeurs sont proches de la neutralité (critère recherché pour un bon substrat de culture). Cependant, les travaux de LANDIs et al. (1990) et LAMHAMEDI et al. (2000) ont montré que le $\mathrm{pH}$ relativement neutre du compost mature à base d'Acacia combiné à la mauvaise qualité de l'eau d'irrigation pouvait affecter négativement la disponibilité des éléments nutritifs dans la rhizosphère des plants. Cet effet sur la disponibilité des éléments nutritifs peut se traduire par l'apparition marquée de symptômes de déficience en micro-éléments, surtout chez les plants résineux et parfois chez les plants feuillus (LAMHAMEDI et al., 2000 ; GogorCENA et al., 2001).

Les résultats du tableau I montrent que la salinité des substrats testés est variable entre 0,84 et $1,19 \mathrm{~g} / \mathrm{l}$. La salinité du substrat issu du double criblage successif sur les mailles 8 x $8 \mathrm{~mm}$ et $12 \times 12 \mathrm{~mm}$ est significativement plus élevée que celle du substrat issu du double criblage successif sur les mailles $12 \times 12 \mathrm{~mm}$ et $8 \times 8 \mathrm{~mm}$. Il est constaté surtout que le problème de salinité qui peut affecter certains plants ne se pose pas pour tous les substrats étudiés. SANCHEZMONEDERo et al. (2004) ont montré que l'augmentation de la CE empêche l'imbibition de l'eau et diminue la germination. Une haute CE peut entraver le développement des plants repiqués (KRATKY, Mishima, 1981 ; HeRRERA et al., 2008). 


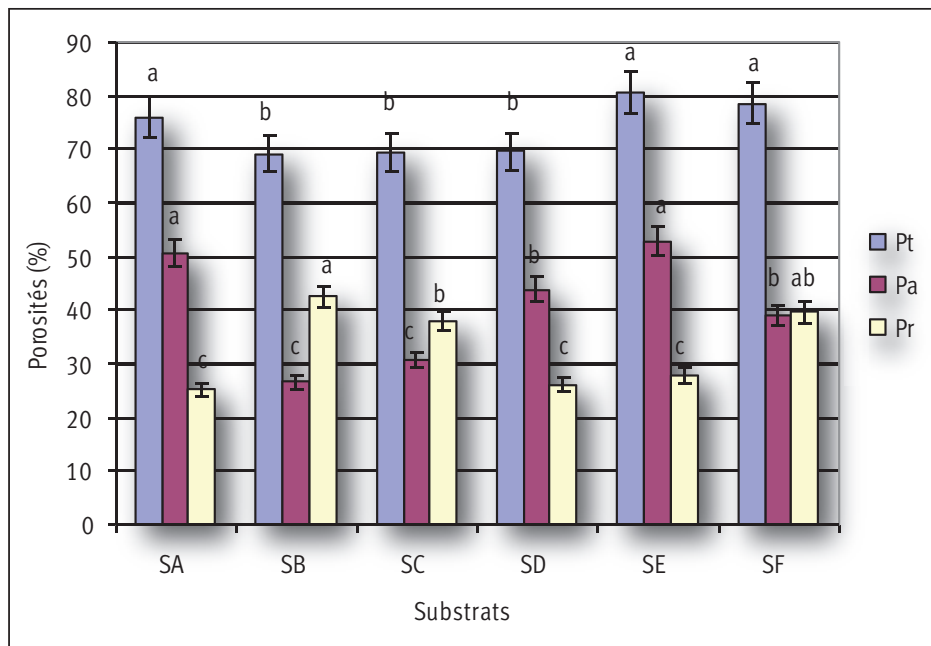

Figure 1.

Porosités totale $(\mathrm{Pt})$, d'aération $(\mathrm{Pa})$ et de rétention $(\mathrm{Pr})$ des substrats de croissance testés.

Les moyennes suivies de la même lettre ne diffèrent pas significativement selon le test de Duncan au seuil de $5 \%$ pour le même paramètre de porosité. Les moyennes de chaque paramètre (barres verticales de même couleur) suivies de lettres différentes indiquent la présence de différences significatives entre les substrats au seuil de $5 \%$ selon le test de Duncan.

Également, en examinant les autres résultats du tableau I, les constatations ci-après peuvent être faites.

- Le taux de matière organique a varié entre 53,6 \% et $64,3 \%$. Ce taux est significativement plus faible chez le substrat issu d'un double criblage successif sur les mailles $12 \times 12 \mathrm{~mm}$ et $8 \times 8 \mathrm{~mm}$ que dans celui issu d'un double criblage successif sur les mailles $8 \times 8 \mathrm{~mm}$ et $12 \times 12 \mathrm{~mm}$.

- Pour les tamisats sylvicoles, le taux de MO est influencé par la taille des particules.

- Le taux de MO le plus bas est relevé dans le cas d'un double criblage sur tamisat (SF).

- Le taux de MO le plus élevé est obtenu dans le cas d'un simple criblage à la grande maille et d'un double criblage successif sur les mailles $8 \times 8 \mathrm{~mm}$ et $12 \times 12 \mathrm{~mm}$ ou criblage sur refus (SD et SE). De tels résultats permettent d'affirmer que l'opération de criblage conduit à une perte de MO au niveau du refus.
- Pour les autres substrats, les taux de MO obtenus sont relativement importants.

- Le rapport $\mathrm{C} / \mathrm{N}$ est largement élevé par rapport aux chiffres cités dans la littérature (C/N variant entre 8 et 15 pour un compost mûr) dans la plupart des cas. Il varie entre 19,1 et 29,0.

- La non-disponibilité de l'azote pour les plants dans le substrat de croissance est l'un des facteurs les plus importants induisant des pertes (GRUDA, SCHNITZLER, 2000).

- Les teneurs des éléments potassium (K) et phosphore (P) sont significativement faibles dans tous les substrats, ce qui justifie l'apport supplémentaire d'une autre source de ces éléments.

L'Acp réalisée a permis de traiter simultanément un certain nombre de paramètres, tous quantitatifs, pour revenir à un espace de dimension réduite plus aisément interprétable. Il est possible alors de représenter les substrats de croissance dans un espace à deux dimensions et d'y observer les proximités entre substrats pouvant s'interpréter comme similarité de comportement chimique (figure 2).

La figure 3 montre que la composante 1 (C1) a une corrélation négative avec les paramètres chimiques $\mathrm{pH}, \mathrm{N}$ et $\mathrm{P}$ (plus C1 est grand, plus les valeurs chimiques sont faibles) et une corrélation positive avec les autres paramètres (S, CE, C/N, MO, COT, K).

La composante 2 (C2) est négativement corrélée avec les paramètres $\mathrm{C} / \mathrm{N}, \mathrm{pH}, \mathrm{MO}, \mathrm{CE}$, $\mathrm{S}$ et $\mathrm{P}$; $\mathrm{C} 2$ est positivement corrélée avec COT, $\mathrm{N}$ et $\mathrm{K}$.

En définitive, les deux facteurs indépendants C1 et C2 représentent les trois quarts de la dispersion des paramètres chimiques considérés.

\section{Résultats relatifs au comportement des plants d'Acacia cyanophylla}

\section{Pourcentage de germination des semences}

La figure 4 illustre les résultats relevés de germination des semences d'Acacia cyanophylla installées au sein de chaque substrat de culture considéré durant les six semaines après semis.

Les pourcentages de germination d'A. cyanophylla sur les différents substrats sont similaires et non significativement différents. La différence est également négligeable durant le suivi, à partir du troisième relevé.

Tableau I.

Paramètres chimiques des différents substrats étudiés.

\begin{tabular}{|c|c|c|c|c|c|c|c|c|c|}
\hline Échantillons & $\mathrm{pH}$ & Conductivité (mmhos $\left./ \mathrm{cm}^{3}\right)$ & Salinité $(g / l)$ & MO (\%) & $\operatorname{Cot}(\%)$ & $\mathrm{N}(\%)$ & $\mathrm{C} / \mathrm{N}$ & $\mathrm{P}(\%)$ & $\mathrm{K}(\%)$ \\
\hline SA & $6,7^{\mathrm{a}}$ & $1,70^{\mathrm{a}}$ & $1,19^{\mathrm{a}}$ & $64,3^{a}$ & $35,7^{a}$ & $1,23^{e}$ & $29,0^{a}$ & $0,03^{c}$ & $0,41^{c}$ \\
\hline SB & $6,0^{e}$ & $1,65^{\mathrm{ab}}$ & $1,15^{\mathrm{ab}}$ & $60,6^{e}$ & $33,6^{e}$ & $1,76^{a}$ & $19,1^{f}$ & $0,04^{b c}$ & $0,46^{b}$ \\
\hline SC & $6,1^{\mathrm{de}}$ & $1,50^{\mathrm{bc}}$ & $1,05^{b c}$ & $61,4^{d}$ & $34,1^{d}$ & $1,46^{b}$ & $23,3^{d}$ & $0,06^{\mathrm{abc}}$ & $0,53^{a}$ \\
\hline SD & $6,3^{a b}$ & $1,40^{c}$ & $0,98^{c}$ & $63,0^{c}$ & $25,0^{c}$ & $1,45^{b}$ & $24,1^{c}$ & $0,08^{a b}$ & $0,36^{e}$ \\
\hline SE & $6,2^{c d}$ & $1,80^{\mathrm{a}}$ & $1,29^{a}$ & $63,8^{b}$ & $35,4^{b}$ & $1,31^{d}$ & $27,0^{b}$ & $0,10^{a}$ & $0,32^{f}$ \\
\hline SF & $6,4^{b}$ & $1,20^{d}$ & $0,84^{d}$ & $53,6^{f}$ & $29,7^{f}$ & $1,37^{c}$ & $21,7^{e}$ & $0,09^{a}$ & $0,39^{d}$ \\
\hline
\end{tabular}

Les moyennes suivies de la même lettre ne diffèrent pas significativement selon le test de Duncan au seuil de $5 \%$. Les moyennes de chaque paramètre (chaque colonne à part) suivies de lettres différentes indiquent la présence de différences significatives entre les substrats au seuil de $5 \%$ selon le test de Duncan. 


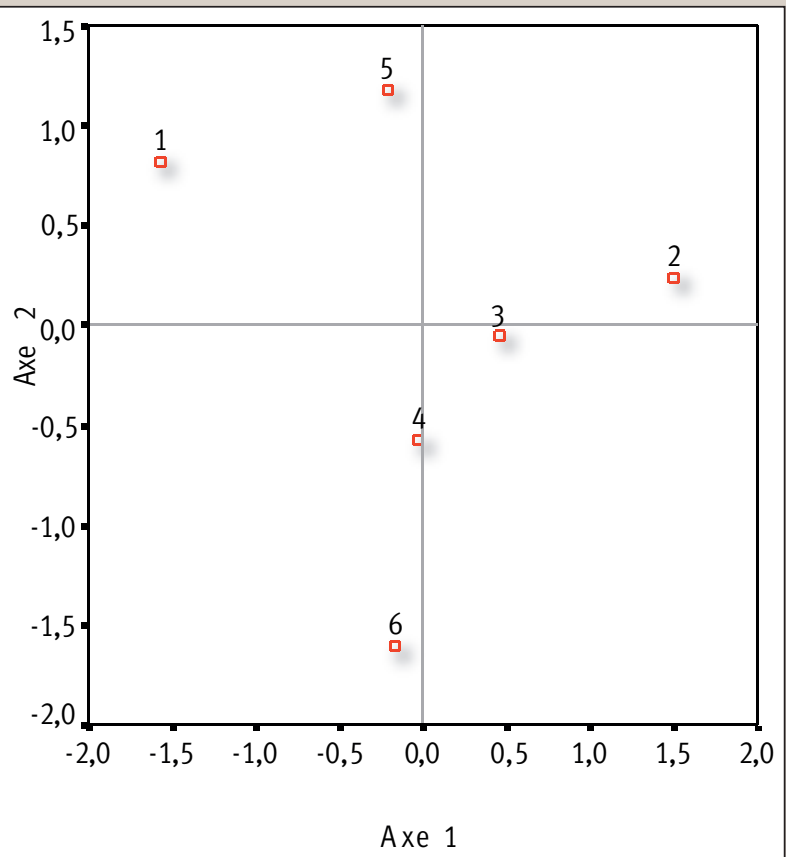

Figure 2.

Représentation des substrats de croissance selon l'analyse en composantes principales. $1,00:$ SA ; $2,00:$ SB ; 3,00:SC ; $4,00: \mathrm{SD} ; 5,00: \mathrm{SE} ; 6,00: \mathrm{SF}$. Les axes 1 et 2 représentent les composantes principales de l'Acp. Cette dernière est responsable de la détermination de deux axes qui expliquent le mieux la dispersion de l'objet, interprété comme un nuage de points. Elle est aussi responsable de l'ordonnancement par inertie expliquée, le second axe étant perpendiculaire au premier.

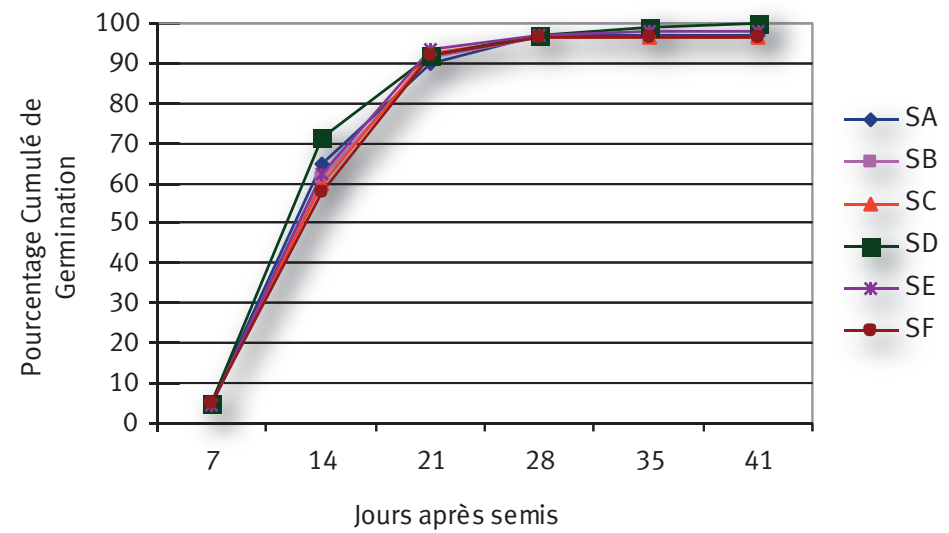

Figure 4.

Pourcentage cumulé de germination des semences d'Acacia cyanophylla.

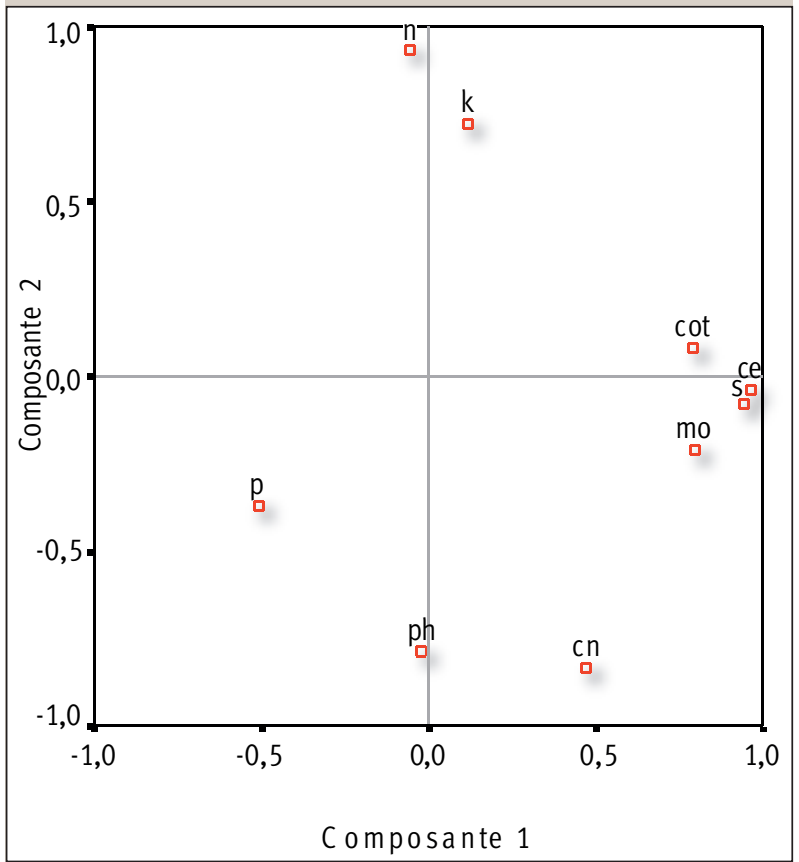

Figure 3.

Représentation des paramètres chimiques des substrats de croissance selon l'analyse en composantes principales. Les composantes 1 et 2 représentent les composantes principales de l'Acp.

\section{Croissance en hauteur et en diamètre des plants}

Les figures 5 et 6 présentent les résultats relevés de croissance des jeunes plants d'A.cyanophylla installés sur chaque substrat de culture étudié durant les trois relevés considérés, respectivement pour la hauteur et le diamètre.

La hauteur et le diamètre sont considérés parmi les facteurs morphologiques qui peuvent prédire au mieux la performance des plants après plantation. La hauteur constitue un bon indicateur de la capacité photosynthétique et de la surface de transpiration, lesquelles sont étroitement corrélées avec le nombre des feuilles (ARMSON, SADREIKA, 1974, cité par LAMHAMEDI et al., 1997). Le diamètre est généralement corrélé avec différents paramètres morphologiques (hauteur, poids sec total, poids sec des racines et poids sec de la partie aérienne). Les plants ayant un gros diamètre possèdent généralement des racines latérales bien développées (RowAN, 1986, cité par LAMHAMEDI et al., 1997), conférant aux plants un meilleur taux de survie après repiquage (HATCHELL, 1986, cité par LAMHAMEDI et al., 1997).

Le deuxième suivi, effectué 40 jours après semis, montre une légère différence en faveur des substrats grossiers issus du double criblage (SE et SF).

Le troisième suivi, accompli 60 jours après semis, confirme les résultats du deuxième suivi consistant à attribuer le critère du bon substrat aux substrats grossiers, et surtout au substrat SF issu du double criblage sur tamisat. 


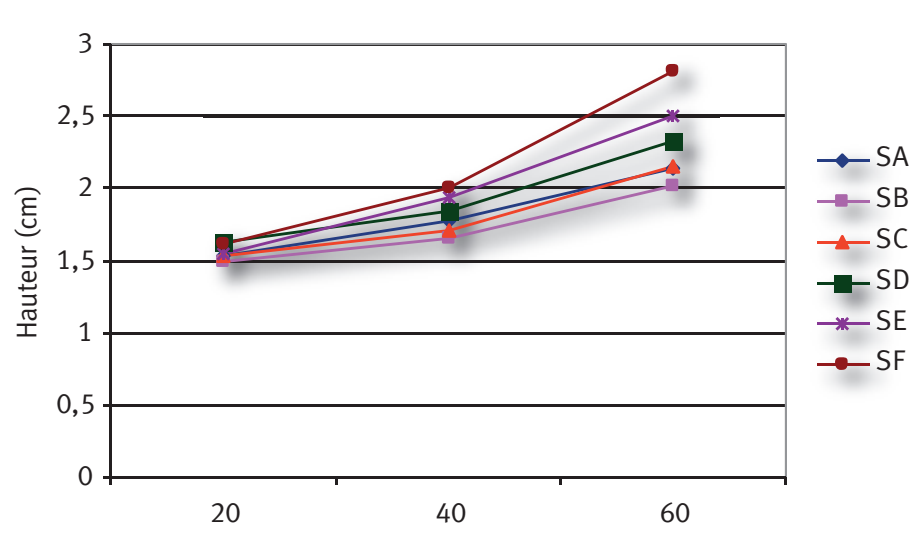

Jours après semis

Figure 5.

Hauteur moyenne (en centimètres) des plants d'Acacia cyanophylla.

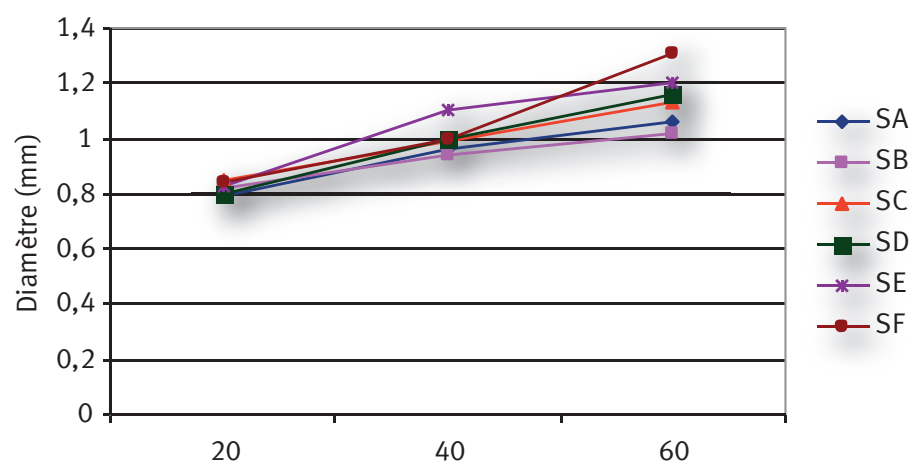

Jours après semis

Figure 6.

Diamètre moyen (en millimètres) des plants d'Acacia cyanophylla.

L'analyse statistique du dernier suivi de la croissance en hauteur des plants ainsi que de leur diamètre, illustrée dans le tableau II, a révélé qu'il existe une différence, certes significative, mais très faible, entre les six substrats de culture testés du point de vue de la hauteur moyenne et du diamètre moyen.

Le substrat SF, issu du double criblage successif sur les mailles $12 \times 12 \mathrm{~mm}$ et $8 \times 8 \mathrm{~mm}$, a enregistré les meilleurs résultats pour les deux paramètres étudiés. Il est suivi du substrat SE, issu du double criblage successif sur les mailles $8 \times 8 \mathrm{~mm}$ et $12 \times 12 \mathrm{~mm}$. Ces résultats sont en accord avec d'autres travaux (M'SADAK et al., $2012 \mathrm{a}, \mathrm{b}$ ).

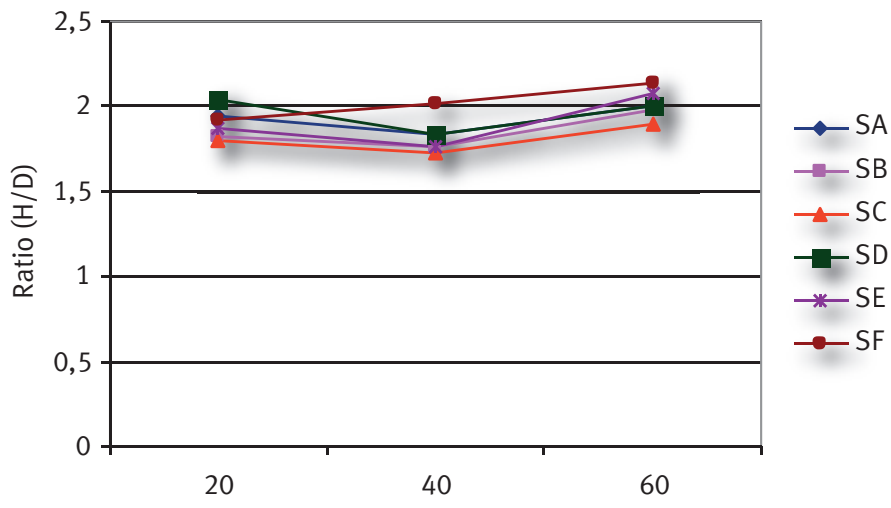

Jours après semis

Figure 7.

Ratio de robustesse des plants d'Acacia cyanophylla.

\section{Ratio de robustesse des plants}

La figure 7 rend compte des résultats obtenus concernant le ratio de robustesse des jeunes plants d' $A$. cyanophylla produits sur chaque substrat de culture étudié durant les trois relevés de croissance mis en œuvre.

Le ratio de robustesse devrait être inférieur à 7 selon les normes (LAMHAMEDI et al., 1997). D'après les résultats obtenus pour le ratio de robustesse, le meilleur substrat est également le substrat SF issu du double criblage sur tamisat, mais il est à noter que ce rapport ne peut pas être un critère de caractérisation immédiate des substrats de croissance, vu que les plants d'A. cyanophylla n'ont pas encore atteint un stade de développement avancé lors du suivi végétatif mis en œuvre.

Tableau II.

Analyse statistique des derniers suivis de la croissance en hauteur et en diamètre des plants.

\begin{tabular}{lll} 
Substrat & HP & DP \\
SA & $2,1^{\mathrm{c}}$ & $1,1^{\mathrm{cd}}$ \\
SB & $2,2^{\mathrm{bc}}$ & $1,1^{\mathrm{d}}$ \\
\hline SC & $2,1^{\mathrm{c}}$ & $1,1^{\mathrm{bcd}}$ \\
SD & $2,3^{\mathrm{bc}}$ & $1,1^{\mathrm{bc}}$ \\
SE & $2,5^{\mathrm{b}}$ & $1,2^{\mathrm{b}}$ \\
SF & $2,8^{\mathrm{a}}$ & $1,3^{\mathrm{a}}$
\end{tabular}

HP : hauteur des plants $(\mathrm{cm})$; DP : diamètre des plants $(\mathrm{mm})$. Les moyennes suivies de la même lettre ne diffèrent pas significativement selon le test de Duncan au seuil de $5 \%$. Les moyennes de chaque paramètre (chaque colonne à part) suivies de lettres différentes indiquent la présence de différences significatives entre les substrats au seuil de $5 \%$ selon le test de Duncan. 
Conclusion

La procédure de criblage du compost sylvicole produit a une influence significative sur son indice de maturité ainsi que sur ses caractéristiques physico-chimiques.

Partant de l'évaluation directe (maturité ; porosités totale, d'aération et de rétention ; paramètres chimiques) des composts et tamisats sylvicoles selon les techniques du simple criblage et du double criblage (selon les deux méthodes : sur refus et sur tamisat) ainsi que de leur évaluation indirecte (comportements germinatif des semences de l'espèce Acacia cyanophylla et végétatif des plants produits en conteneurs), il est possible d'affirmer que le meilleur substrat est le tamisat issu du double criblage sur les mailles carrées $12 \times 12 \mathrm{~mm}$ et $8 \times 8 \mathrm{~mm}$ dans les conditions expérimentales adoptées ; cependant, le recours à un tamisat issu du simple criblage est fortement préconisé en raison d'un ajustement granulométrique obtenu avec un gain occasionné de temps et d'argent. À ce propos, en tenant compte des résultats obtenus directement et indirectement pour les tamisats issus des mailles $8 \times 8 \mathrm{~mm}$ et $12 \times 12 \mathrm{~mm}$, il convient de tester, dans l'avenir, la maille $10 \times 10 \mathrm{~mm}$, pouvant convenir le mieux au criblage à mettre en œuvre.

En définitive, il convient de signaler que le paramètre morphologique ratio de robustesse est à considérer avec prudence, étant donné que le suivi a été limité à 60 jours après semis. Une telle période est insuffisante pour apprécier ce paramètre. Ce dernier est communément mieux apprécié à la fin du cycle de production des plants forestiers, aspect méritant d'être exploré lors d'investigations futures. En outre, un tel paramètre reste plus important pour le forestier que la hauteur et le diamètre, étant donné qu’il lie ces derniers. Une telle constatation amène à examiner les différences significatives relevées dans le contexte expérimental considéré avec une certaine précaution.
ALSANIUS B., JENSEN P., ASP H. (éds), 2004. Proceedings of the International Symposium on Growing Media and Hydroponics. Louvain, Belgique, International Society for Horticultural Science, Acta Horticulturae 64, 644 p.

BILDERBACK T. E., FONTENO W. C., JOHNSON D. R., 1982. Physical properties of media composted of peanut hulls, pine bark, and peat moss and their effects on azalea growth. Journal of the American Society for Horticultural Science, 107: 522-525.

BUGBEE G. J., FRINK C. R., 1986. Aeration of potting media and plant growth. Soil Science, 141: 438-441.

FITZPATRICK G. E., 2001. Compost utilization in ornamental and nursery crop production systems. In: Compost utilization in horticultural cropping systems. Stoffella P. J., Kahn B. A. (éds). New York, États-Unis, Lewis Publishers, p. 135-150.

GOGORCENA Y., MOLIAS N., LARBI A., ABADIA J., ABADIA A., 2001. Characterization of the responses of cork oak (Quercus suber) to iron deficiency. Tree Physiology, 21: 1335-1340.

GOYAL S., DHULL S. K., KAPOOR K. K., 2005. Chemical and biological changes during composting of different organic wastes and assessment of compost maturity. Bioresource Technology, 96: 1584-1591.

GRUDA N., SCHNITZLER W. H., 2000. The effect of water supply on biomorphological and plant-physiological parameters of tomato transplants cultivated in wood fiber substrate. Journal of Applied Botany/Angewandte Botanik, 74: 233-239.

GUEHL J. M., FALCONNET G., GRUEZ J., 1989. Caractéristiques physiologiques et survie après plantation de plants de Cedrus atlantica élevés en conteneurs sur différents types de substrats de culture. Annales des Sciences Forestières, 46 (1) : 1-14.

HAUG R. T., 1993. Practical handbook of compost engineering. Boca Raton, FL, États-Unis, Lewis Publishers, 441 p.

HERRERA F., CASTILLO J. E., CHICA A. F., LOPEZ BELLIDO L., 2008. Use of municipal solid waste compost (MSWC) as a growing medium in the nursery production of tomato plants. Bioresource Technology, 99: 287-296.

ISO, 1994. Qualité du sol: Détermination du pH et de la CE spécifique. Genève, Suisse, International Standardisation Organisation, $4 \mathrm{p}$.

KRATKY B. A., MISHIMA H. Y., 1981. Lettuce seedling and yield response to preplant and foliar fertilization during transplant production. Journal of the American Society for Horticultural Science, 106 (1): 3-7.

LAMHAMEDI M. S., FORTIN J. A., 1994. La qualité des plants forestiers : critères d'évaluation et performance dans les sites de reboisement. In : Abourouh M. (éd.). Actes de la première journée nationale sur les plants forestiers. Rabat, Maroc, Centre de recherche et d'expérimentation forestières, p. 35-55. 
LAMHAMEDI M. S., FORTIN J. A., AMMARI Y., BEN JELLOUN S., POIRIER M., FECTEAU B., BOUGACHA A., GODIN L., 1997. Évaluation des composts, des substrats et de la qualité des plants élevés en conteneurs. Tunis, Tunisie, Direction générale des forêts et Pampev International, Projet Bird $n^{\circ} 3601$.

LAMHAMEDI M. S., AMMARI Y., FECTEAU B., FORTIN J. A., MARGOLIS H., 2000. Problématique des pépinières forestières en Afrique du Nord et stratégies d'orientation. Cahiers Agricultures, 9 (5) : 369-380.

LANDIS T. D., TINUS R. W., MC DONALD S. E., BARNETT J. P., 1990. The Container Tree Nursery Manual. Vol. 4: Seedling nutrition and irrigation. Agriculture Handbook Series, 674. Washington, DC, États-Unis, US Department of Agriculture, Forest Service, $119 \mathrm{p}$.

LANDIS T. D., 1990. The Container Tree Nursery Manual. Vol. 2: Containers and growing media. Agriculture Handbook Series, 674. Washington, DC, États-Unis, US Department of Agriculture, Forest Service, 41-85.

LEMAIRE F., DARTIGUE A., RIVIÈRE L. M., CHARPENTIER S., 1989. Culture en pots et conteneurs. Principes agronomiques et applications. Paris, France, Inra, $181 \mathrm{p}$.

MILLER J. H., JONES N., 1995. Organic and compost-based growing media for tree seedlings nurseries. Washington, DC, États-Unis, Banque mondiale, World Bank Technical Papers, 264, $75 \mathrm{p}$.

M'SADAK Y., ELOUAER M. A., SAAD H., 2012 a. Évaluation comparée du comportement des plants d'Acacia cyanophylla installés sur différents substrats de croissance. Communication par affiche. Forum international Atsb, 21-24 mars 2012, Hammamet, Tunisie.

M'SADAK Y., ELOUAER M. A., SAAD H., 2012 b. Comportement comparé des plants d'Eucalyptus gompho produits sur différents substrats de culture. Communication par affiche. Colloque international Qpe-Tvr, 20-22 avril 2012, Mahdia, Tunisie. NKONGOLO N. V., CARON J., 1999. Bark particle sizes and the modification of the physical properties of peat substrates. Canadian Journal of Soil Science, 79: 111-116.

RAVIV M., ZAIDMAN B. Z., KAPULNIK Y., 1998. The use of compost as a peat substitute for organic vegetable transplants production. Compost Science \& Utilization, 6 (l): 46-52.

REISCH K. W., 1967. Rooting media. Proceedings of the International Plant Propagators' Society, 117: 356-361.

RICHARDS D., LANE M., BEARDSELL D. V., 1986. The influence of particle-size distribution in pinebark:sand:brown coal potting mixes on water supply, aeration and plant growth. Scientia Horticulturae, 29: 1-14.

ROSE R., HAASE D. L., BOYER D., 1995. Organic matter management in forest tree nurseries: theory and practice. Corvallis, OR, États-Unis, Nursery Technology Cooperative, Oregon State University, $67 \mathrm{p}$.
SANCHEZ-MONEDERO M., ROIG A., CEGARRA J., BERNAL M. P., NOGUERA P., ABAD M., ANTON A., 2004. Composts as media constituents for vegetable transplant production. Compost Science \& Utilization, 12 (2): 161-168.

STOFFELLA P. J., KAHN B. A., 2001. Compost utilization in horticultural cropping systems. New York, États-Unis, Lewis Publishers, $413 \mathrm{p}$.

TILT K. M., BILDERBACK T. E., FONTENO W. C., 1987. Particle size and container size effects on growth of three woody ornamental species. Journal of the American Society for Horticultural Science, 112: 981-984.

TIQUIA S. M., 2010. Reduction of compost phytotoxicity during the process of decomposition. Chemosphere, 79: 506-512.

VERDONCK O., PENNINCK R., DE BOOT M., 1983. Physical properties of different horticultural substrates. Acta Horticulturae, 150: 155-160. 